\title{
Modelo integrado de gestión humana y del conocimiento: una tecnología de aplicación
}

\author{
Cuesta Santos, Armando*
}

\section{Resumen}

El objetivo de este artículo es diseñar un modelo integrado de gestión humana (RRHH) y del conocimiento, bajo un enfoque o perspectiva estratégica. La metodología de investigación implicó la investigación-acción, mediante la intervención del autor a través de la maestría sobre gestión de recursos humanos en distintas empresas cubanas. Como resultado fundamental se alcanzó una tecnología para la aplicación práctica del Modelo Integrado de Gestión de Recursos Humanos, comprendiendo el diagnóstico, la planificación y el control estratégico del desempeño de esa gestión, que tuvo un aval práctico en el país. Como conclusión se obtiene el modelo propuesto que difiere de los reflejados en la literatura científica actual al considerar el ciclo completo de la gestión del cambio. El valor agregado esencial lo ofrece la generación de una tecnología, para la aplicación del modelo además de la consideración integracionista de la gestión de recursos humanos con la gestión del conocimiento.

Palabras clave: Gestión de recursos humanos, gestión del conocimiento, gestión humana, normas cubanas.

\section{Integrated Human and Knowledge Management Model: An Application Technology}

\begin{abstract}
The objective of this study was to design an integrated model for human resource and knowledge management using a strategic approach or perspective. The methodology implied action research during studies for a master's degree on human resource management in diverse Cuban businesses. A fundamental result was technology for practical application of the Integrated Model for Human Resource Management; the model includes diagnosis, planning and strategic control of that management, which received practical approval in the country. In conclusion, the proposed model
\end{abstract}

Recibido: 10-01-11. Aceptado: 09-11-11

* $\quad$ Psicólogo. Master en Organización del Trabajo. Doctor en Ciencias Económicas (Ph. D.). Doctor en Ciencias (Dr. Cs.). Profesor Titular de la Facultad de Ingeniería Industrial de la Universidad Tecnológica de La Habana (CUJAE). e-mail: cuesta@ind.cujae.edu.cu 
was obtained, differing from those reflected in current scientific literature in that it considers the complete cycle for managing change. The essential added value is the generation of technology for applying the model in addition to integrating human resource management with knowledege management.

Keywords: Human resource management, knowledge management, human management, $\mathrm{Cu}-$ ban standards.

\section{Introducción}

En la década de 1990, en el ámbito de la gestión en países desarrollados, se enfatizó la recurrencia a modelos conceptuales de gestión humana o de gestión de recursos humanos (RRHH) en las empresas (Senge, 1999; Werther y Davis, 2001; Chiavenato, 2002; Ulrich, 2006). Y en esa década se acuñó en ese mismo ámbito, la gestión del conocimiento (Edvinsson y Malone, 1999; Gates, 1999; Sveiby, 2000). Esta década signó el desarrollo de la gestión humana estratégica (Lengnick-Hall et al., 2009; Boxall et al., 2007) en su vínculo con la estructura y componentes de sistemas de $\mathrm{RRHH}$ con sus correspondientes modelos conceptuales.

En la primera década del siglo XXI, la recurrencia a esos modelos comienza a destacar en países de economías emergentes o en vías de desarrollo como en China, Brasil y la India (Wey y Lau, 2005; Fleury y Fleury, 2005; Som, 2007), así como en Cuba (Cuesta, 2005; NC 3001: 2007; Morales, 2009).

A fines del siglo XX, el impacto de las nuevas Tecnologías de la Información y las Comunicaciones (TIC) en la gestión de RRHH implicaron los e-RRHH (Cuesta, Alcaide y López, 2010), aún incipientes en la práctica empresarial. Ese impacto de las TIC, desde su surgimiento centrado en la administración de personal
(Fitz-eng, 1999; Gates, 1999; Dolan et al., 2003), buscó sistematizar de modo automatizado las características de los recursos humanos o del capital humano; pero comprendió más al sistematizar la información relativa a estructuras organizativas, relaciones con clientes, procedimientos organizativos y know how organizacional derivado del accionar del capital humano: el capital estructural era considerado en esos sistemas. Las nuevas TIC indujeron la amplitud del objeto de la gestión de RRHH hacia la gestión del conocimiento. El tratamiento del capital intelectual es inmanente hoy a los e-RRHH.

Interesa, sin dudas, al mundo de la gestión laboral, considerar modelos conceptuales que comprendan de manera integrada la gestión humana o de RRHH del conocimiento desde una perspectiva estratégica. Es fundamental enfocar de manera holística esa gestión humana junto a la gestión del conocimiento, respondiendo o alineándose a la estrategia organizacional, o lo que es igual, integrando esa gestión a la estrategia.

El objetivo de este artículo es diseñar un modelo integrado de gestión humana y del conocimiento, que a su vez se integra a la estrategia organizacional, con una tecnología para su aplicación práctica comprendiendo el diagnóstico, la proyección (planificación) y el control estratégico del desempeño de esa gestión, que tuvo un aval práctico en el país. Este 
modelo es un producto que se derivo de manera esencial que se derivó de manera esencial de la dinámica de trabajo generada en la Maestría en Gestión de Recursos Humanos (GRH) impartida al Consejo de Dirección del Ministerio del Trabajo y Seguridad Social de Cuba. Dicho modelo considera la norma cubana (NC) sobre "gestión del capital humano" (NC 3001:2007). La metodología de trabajo investigativo que se empleó fue la de observación participante -desde la condición de profesor y coordinador de la Maestría en GRH del autor de este artículo-, siendo interventora la impartición de esa Maestría en GRH.

\section{Conceptualización del modelo de RRHH DPC y sus enfoques}

Antecedente del modelo que se propone, lo constituyó el decursar de la Maestría en GRH desarrollada por la Facultad de Ingeniería Industrial de la Universidad Tecnológica de La Habana (CUJAE), iniciada en 1994 (Cuesta, Viña y Marsán, 1994). El primer modelo referente fue el de William B. Werther y Keith Davis de la Universidad de la Florida (Werther y Davis, 2001), con posterioridad se recurrió al de Michael Beer y colaboradores de la Universidad de Harvard (Beer et al., 1985).

El modelo de RRHH de Beer y colaboradores de la Harvard Business School, modificado por este autor al incluirle la Auditoria, junto a la inclusión de otras preguntas clave, así como indicadores y técnicas asociados a las mismas, llegando a configurar una "tecnología para el diagnóstico y la proyección de la
$R R H H^{\prime \prime},($ Cuesta, 1999) se utilizó durante varios años.

En la Maestría en RRHH de la CUJAE, desde 1997 se orientó la aplicación de ese modelo modificado. Más de un centenar de especialistas y directivos -del país y del extranjero- lo utilizaron desde 1997 en sus proyectos de curso y tesis de maestría, contribuyendo con sus experiencias prácticas en las empresas (Aguirre, 2001; Hernández, 2003; Ballivián, 2006; Sánchez, 2007) a perfilar y mejorar ese modelo de RRHH, y en particular, la concebida tecnología de diagnóstico y proyección expresa en la primera edición del libro Tecnología de gestión de recursos humanos (Cuesta, 1999), y con posterioridad, en la tecnología del modelo RRHH DPC expresa en su segunda edición (Cuesta, 2005).

Los enfoques que son inmanentes al modelo son los siguientes: sistémico, estratégico, participativo, interdisciplinario, de procesos y de competencias laborales (Cuesta, 2005 y 2010).

El enfoque en sistema significa que la gestión de RRHH es más que la suma simple de sus partes o procesos clave, es una nueva cualidad surgida de la interacción de sus partes, es una integralidad. Lo sistémico aquí rechaza el enfoque tayloriano, parcelado y especializado que mutila al empleado su potencial polivalente (multicompetencias), devenido en sustento de los sistemas de trabajo flexibles. El enfoque estratégico señala la proactividad, la actuación anticipada, contraria a la reactiva caracterizada por accionar cuando se presenta el problema o la dificultad, o peor aún, después de su manifestación; a la estrategia se alinea el accionar del modelo RRHH DPC. Lo in- 
terdisciplinario indica que la RRHH demanda la acción de diferentes disciplinas científicas, atendiendo a lo complejo no lineal, errando quien pretenda sesgarla con el predominio de alguna disciplina en esa totalidad; a la vez indica su accionar transdisciplinario o transfuncional, respecto al resto de las áreas funcionales. El enfoque participativo comprende la cada vez más creciente influencia de los empleados en las actividades de RRHH y de la organización toda, y en especial en la toma de decisiones; y ello lo materializa la recurrencia a técnicas participativas. El enfoque de procesos centrado en el cliente, tanto interno como externo, se caracteriza por considerar la cadena de creación del nuevo valor agregado desde el proveedor hasta el cliente. $Y$ el enfoque por competencias laborales se caracteriza porque la gestión de RRHH se concentra en gestionar considerando las competencias laborales que portan las personas que trabajan.

Se refleja en el Diagrama 1 el modelo de RRHH DPC. Para otorgarle una identificación a ese modelo referente de $\mathrm{RRHH}$, se le denominó modelo de RRHH DPC, significando esas siglas: diagnóstico $(D)$, proyección $(P)$ y control de gestión estratégica (C). Sería: Modelo de Gestión de Recursos Humanos de Diagnóstico, Proyección y Control de gestión estratégica. Así, su nombre indica su funcionalidad, acarreando la aplicación de su implícita tecnología la action-research, en la acepción de Kurt Lewin (1948), de modo que a la vez que en el proceso de investigación se va diagnosticando o interpretando, se va proyectando, para inmediatamente después ejecutar o transformar según lo proyectado, y con posterioridad se va a controlar (feedback) esa ejecución o gestión consecuente con la estrategia organizacional.

Es importante subrayar que, no obstante la experiencia positiva que condujo al modelo de RRHH DPC, se defiende la idea de la necesidad de configurar modelos que respondan al "traje a la medida" que precisa la empresa u organización laboral en cuestión. Por ello se le considera como un modelo referente.

Por la experiencia adquirida se defiende esa autóctona cualidad del "traje a la medida" o ad hoc que precisa la empresa u organización laboral, con mucho acierto planteado en el desarrollo del proceso de perfeccionamiento empresarial cubano (Tristá, 2002). Aunque en este modelo se destacan invariantes de esta contemporaneidad (especialmente las competencias laborales y la Learning Organization: pilares de la gestión del conocimiento), las mismas están sujetas necesariamente a la dialéctica, especialmente de manera inmediata sus indicadores y técnicas.

El modelo de RRHH DPC tiene en el centro de sus subsistemas y políticas de RRHH a la persona manifiesta en su educación y desarrollo. La persona, su educación y desarrollo humano, es el fin y no el medio. Tal educación y desarrollo es el referente obligado para los restantes subsistemas y políticas (como refleja el Diagrama 2), donde quedarán incluidos todos los procesos o actividades clave de RRHH en los cuatro subsistemas que se reflejan en el bloque de la figura 1 denominado "Subsistemas y políticas de RRHH": 


\section{Diagrama 1}

Modelo conceptual de gestión de recursos humanos, de diagnóstico, proyección y control de gestión estratégica

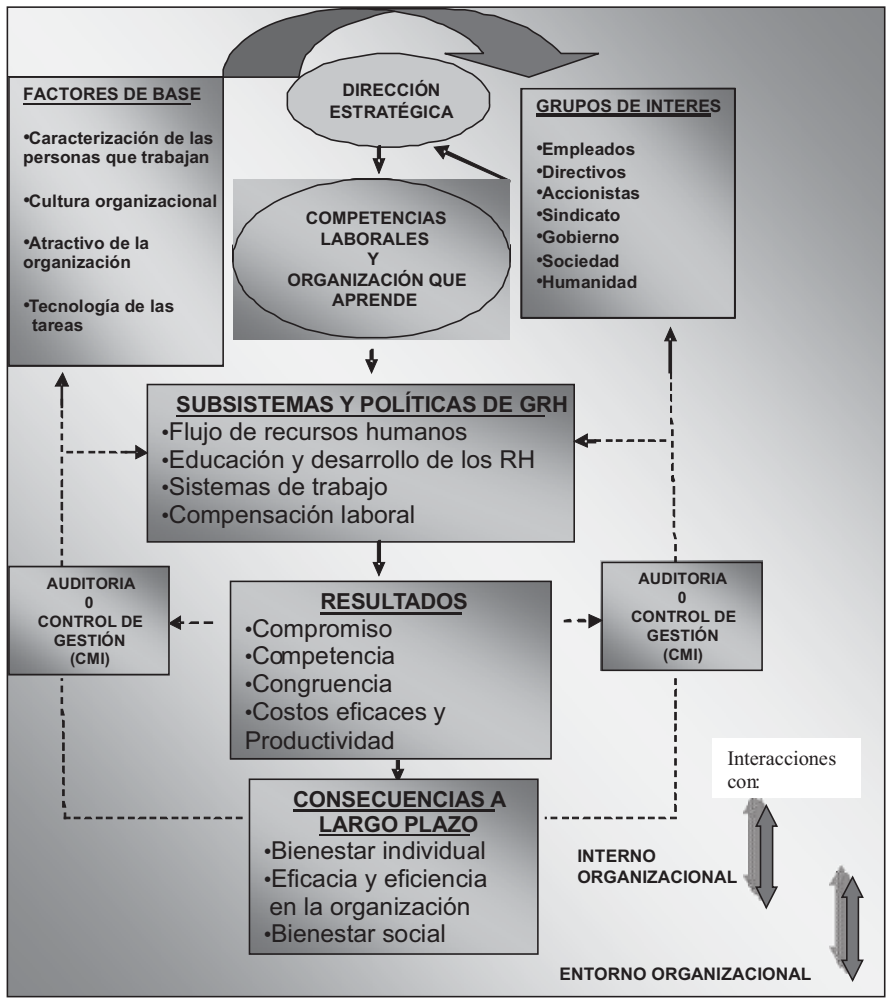

Fuente: Cuesta (2005).

- Flujo de recursos humanos: inventario de personal, reclutamiento y selección de personal, colocación, evaluación del desempeño, evaluación del potencial humano, promoción, democión, jubilación, recolocación o outplacement.

- Educación y desarrollo: formación, planes de carrera, planes de comunicación, organización que aprende, participación, desempeño de cargos y tareas, planes de las nuevas TIC (recurrencia a Internet, Intranet, sistemas de información sobre $\mathrm{RRHH}, \quad$ $R R H H$, e-selección, e-learning, etc.).

- Sistemas de trabajo: organización del trabajo, seguridad e higiene ocupacional, ergonomía, planificación de recursos humanos y optimización de plantillas, perfiles de cargo.

- Compensación laboral: sistemas de estimulación material y moral, sistemas de pago a tiempo y por rendimiento, sistemas de reconocimiento social, sistemas de motivación, evaluación de puestos, entre otros. 


\section{Diagrama 2 \\ Subsistemas y políticas del modelo de gestión de recursos humanos, de diagnóstico, proyección y control de gestión estratégica}

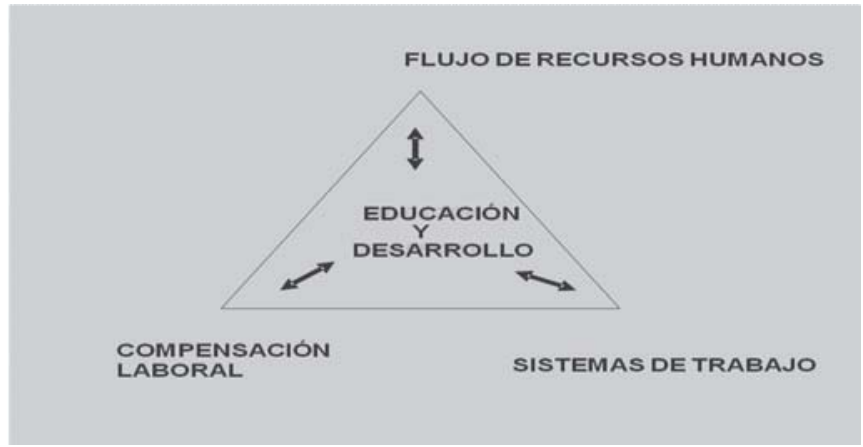

Fuente: Cuesta (2005).

A la vez, esas cuatro denominaciones son las mismas para cada una de las cuatro políticas que regirán las agrupaciones de todos los procesos clave que sean considerados; así se evita la dispersión en el accionar sobre los recursos humanos. Esa concentración contribuye a la síntesis necesaria y al enfoque sistémico.

Para Cuba, de trabajarse en la acreditación o aplicación por las Normas Cubanas (NC) 3000, 3001 y 3002: (2007), se asumirían los nueve (9) módulos o procesos clave en el cuadro de "Subsistemas y políticas del modelo de RRHHDPC". Y de los "Requisitos" que en esas NC se consignan, originarían las preguntas clave a responder.

El modelo de RRHH DPC, reflejado en el Diagrama 1, parte de los conceptos contemplados en el bloque "Factores de base”. Significan el ser social, las condiciones objetivas. Respecto a las manifestaciones de los "Grupos de interés" (aspiraciones, actitudes, motivaciones, intereses), los factores de base son sus determinantes, y así lo indica la lucha. $Y$ son esas manifestaciones de los grupos de interés, las que determinarán la "dirección estratégica”, liderada por los directivos.

La relevancia significativa para los sistemas de compensación laboral en ese modelo funcional, se aprecia en la consideración de la "tecnología de las tareas", tanto en su acepción hard como soft (donde son implicados los procesos de trabajo), puesto que se concibe a la tecnología de las tareas como determinante de la compensación laboral y pilar tecnológico del sistema RRHH. Es uno de los procesos decisivos entre los "Factores de base".

Es muy importante advertir que los sistemas de trabajo y logístico, concebidos hoy a la luz de los aportes de la reingeniería como conjuntos de procesos de trabajo, se consideran pilares tecnológicos de la gestión de RRHH, y por eso están concebidos bajo el concepto "tecnología de las tareas". Solamente después que tales procesos de trabajo sean bien definidos, entran los sistemas de com- 
pensación que le complementen. En no pocas ocasiones ha ocurrido que se ha empezado por el final y no por el principio. La remuneración salarial y la compensación en general, técnicamente deben generarse como resultante del diseño de los sistemas de trabajo y logístico, y no al revés.

Los "grupos de interés" comprenden esencialmente los indicados en el modelo. Ahí el grupo de interés humanidad es la categoría suprema. Trasciende al grupo sociedad, que bien puede referirse a un país o región. Considerar las manifestaciones de las contradicciones entre esos grupos, resulta especialmente importante; recuérdese que el desarrollo viene dado por el carácter contradictorio de la realidad: la ley de la unidad y lucha de contrarios aquí suele reflejarse con fuerza. Ellos determinan a la "dirección estratégica" que ocupa el lugar cimero en el modelo, en tanto su orientadora y aglutinadora sinergia. $Y$ todo eso pasa con inmediatez por el bloque del modelo titulado "Competencias laborales y organización que aprende", significando ambos elementos un subconjunto peculiar, rector, en el modelo. En esa "dirección estratégica", además, van comprendidas las orientaciones políticas y de gobierno.

$\mathrm{Si}$ se asume la tesis que afirma que la ventaja competitiva básica de las empresas en el mundo globalizado del porvenir, en el mundo de inicios del siglo XXI, no radicará en sus recursos materiales, ni en especifico en los recursos energéticos, recursos financieros, y ni en la tecnología, sino que la ventaja competitiva básica de las empresas a inicios del nuevo milenio radicará en el nivel de formación (competencia) y gestión de sus recursos humanos. Y a la vez, si se comprende bien que de esos dos elementos, formación y gestión, el primero es el determinante, se inferirá claramente la importancia de considerar a las competencias laborales que portan las personas. Y como esas "competencias" no se dan de una vez y para siempre, y hay que mantenerlas vitales y en continuo desarrollo, se impone un tipo de organización que garantice su continua renovación: una "organización que aprende".

Necesario es precisar que, si bien en ese modelo la educación y desarrollo es el centro y referente obligado para el resto de los subsistemas y políticas, el sistema de trabajo es su base fundamental con los procesos de trabajo implicados. El diseño o rediseño de procesos de trabajo, concebido como espiral dialéctica de perfeccionamiento empresarial o mejoramiento continuo en aras de la calidad requerida, es definitorio en la eficacia de la gestión de RRHH.

Después del cuadro o bloque relativo a "Subsistemas y políticas de RRHH", corresponde el concerniente a "Resultados", donde el resultado compromiso con la organización se considera el de mayor importancia. Le sigue el bloque "Consecuencias a largo plazo", y después procede la retroalimentación del sistema, como indican las flechas, dada por los resultados de la "Auditoria o control de gestión (CMI)". Se retroalimentan los "Factores de base" y los "Grupos de interés", que son los dos bloques de inicio, y también los subsistemas y políticas de RRHH, los más susceptibles de forma inmediata al cambio.

Como se señala en el modelo RRHHDPC, esa actividad antes reseñada se halla en interacción con el "interno or- 
ganizacional" (el resto de las áreas o procesos funcionales de la empresa, tales como finanzas, producción, ventas, etc.) y el "entorno organizacional". Y el mismo ejerce una funcionalidad sobre ellos.

\section{La tecnología del modelo RRHH DPC}

La tecnología de diagnóstico, proyección y control de gestión estratégica de la gestión de $R R H H$ implicada por este modelo considerando la conducción metodológica antes enunciada, comprende la siguiente lógica: conceptos, preguntas clave $(P C)$, indicadores y técnicas para su aterrizaje. Esa tecnología comprende el ciclo de diagnostico, planeación, implantación y control de la gestión de $\mathrm{RRHH}$ acorde con la estrategia trazada.

Ese control estratégico técnicamente se identifica con el Cuadro de Mando Integral (CMI) argumentado por Robert Kaplan y David Norton (Kaplan y Norton, 2004). El modelo propuesto por esos autores, que ellos mismos consideraron modelo de gestión del conocimiento, se inserta muy coherentemente en esta tecnología, condicionando la planificación, en la cual se defiende con particular énfasis un conjunto de indicadores intangibles, habiendo similitudes con los defendidos por los gestores de Skandia, Leif Edvinsson y Michael Malone (1999).

De inmediato para ilustrar, se indica el conjunto de conceptos y preguntas clave $(P C)$ relativas al cuadro o bloque "Factores de base", y seguidamente se enuncian indicadores y técnicas respecto al concepto "Características de las personas que trabajan" (Cuesta, 2005 y 2010):
- Características de las personas que trabajan

PC: ¿Cuál es la situación de vida material de las personas en el trabajo?

PC: ¿Cuál es la naturaleza psicológica de las personas en el trabajo?

- Cultura organizacional

PC: ¿Cuáles son las creencias implícitas o explícitas clave de los directivos acerca de la naturaleza de la organización, su papel en la sociedad y cómo deberá ser dirigida?

PC: ¿Cómo deberán ser tratados y utilizados los empleados de la organización?

- Atractivo de la organización

PC: ¿Qué capacidad de atractivo posee la organización laboral para los empleados actuales y futuros, y cómo se anticipa a las tendencias del entorno del cual obtiene sus personas?

- Tecnología de las tareas

PC: ¿Cómo organizar el trabajo del personal junto a su seguridad e higiene?

PC: ¿Cómo organizar la producción, el aprovisionamiento y la distribución?

- Leyes y valores de la sociedad

PC: ¿Cuáles son las características de la cultura nacional e ideología predominantes?

Los indicadores: nivel (deficiencia) de la gestión de RRHH en la empresa, percepción de perspectivas en la institución, sentimiento de compromiso o pertenencia a la organización, condiciones de vivienda, condiciones laborales, nivel (percepción) de liderazgo, entre otros. Las técnicas más recurridas asociadas a ese concepto: Diagrama Ishikawa Ponderado, Escalón de perspectivas, socio- 
metría, encuesta Likert, encuesta del potencial humano.

Idéntica lógica considerando concepto, preguntas clave, indicadores y técnicas, caracteriza la tecnología al comprender a los bloques de conceptos que continúan: "Grupos de interés", "Dirección estratégica", "Competencias laborales y organización que aprende", "Subsistemas y políticas de RRHH", "Resultados", "Auditoria o control de gestión (CMI)", "Consecuencias a largo plazo", "Interno organizacional" y "Entorno organizacional".

Para Cuba, como antes se apuntó, de trabajarse con fines de acreditación por las Normas Cubanas NC 3000-3002 (2007), se acudiría a considerar las PC que significarían los distintos "Requisitos" en la NC 3001 (2007), cuyos módulos o procesos clave se reflejan en el Diagrama 3. No se contradicen con las PC que comprenden los conceptos del modelo
RRHH DPC, que técnicamente las complementan. El libro que comprendió el modelo RRHH DPC (Cuesta, 2005), fue referencia bibliográfica en la NC 3001 (2007), cumpliendo con la misión o valor de referente que se concedió a ese modelo desde su concepción inicial. El Diagrama 4 resume la referida tecnología.

\section{Conclusiones}

La consideración de modelos conceptuales de gestión humana o de gestión de recursos humanos con enfoque o perspectiva estratégica, es de relevancia práctica en la gestión empresarial de esta contemporaneidad, con independencia del país del cual se trate, particularmente si se considera el contexto de la globalización que impone aumentar los niveles de competitividad.

La recién acuñada gestión del conocimiento de la década de 1990 junto a

\section{Diagrama 3 \\ Esquema del modelo cubano para el diseño e implantación de un "Sistema de Gestión Integrada de Capital Humano", según la NC 3001: 2007}

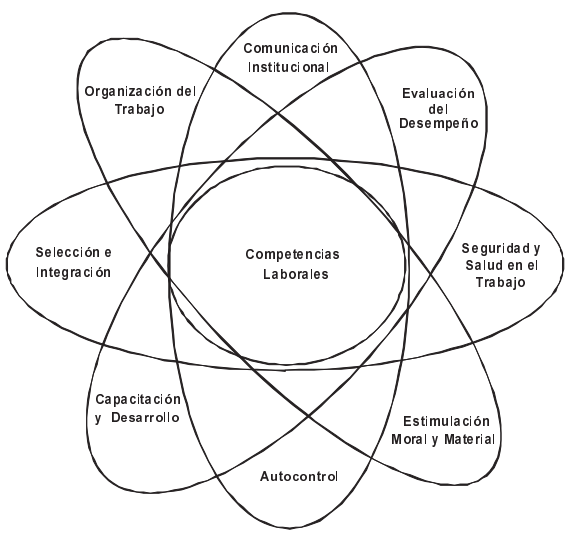

Fuente: Oficina de Normalización de Cuba, 2007. 


\section{Diagrama 4 \\ Tecnología de diagnóstico, proyección (planeación) y control estratégico de la gestión de recursos humanos}

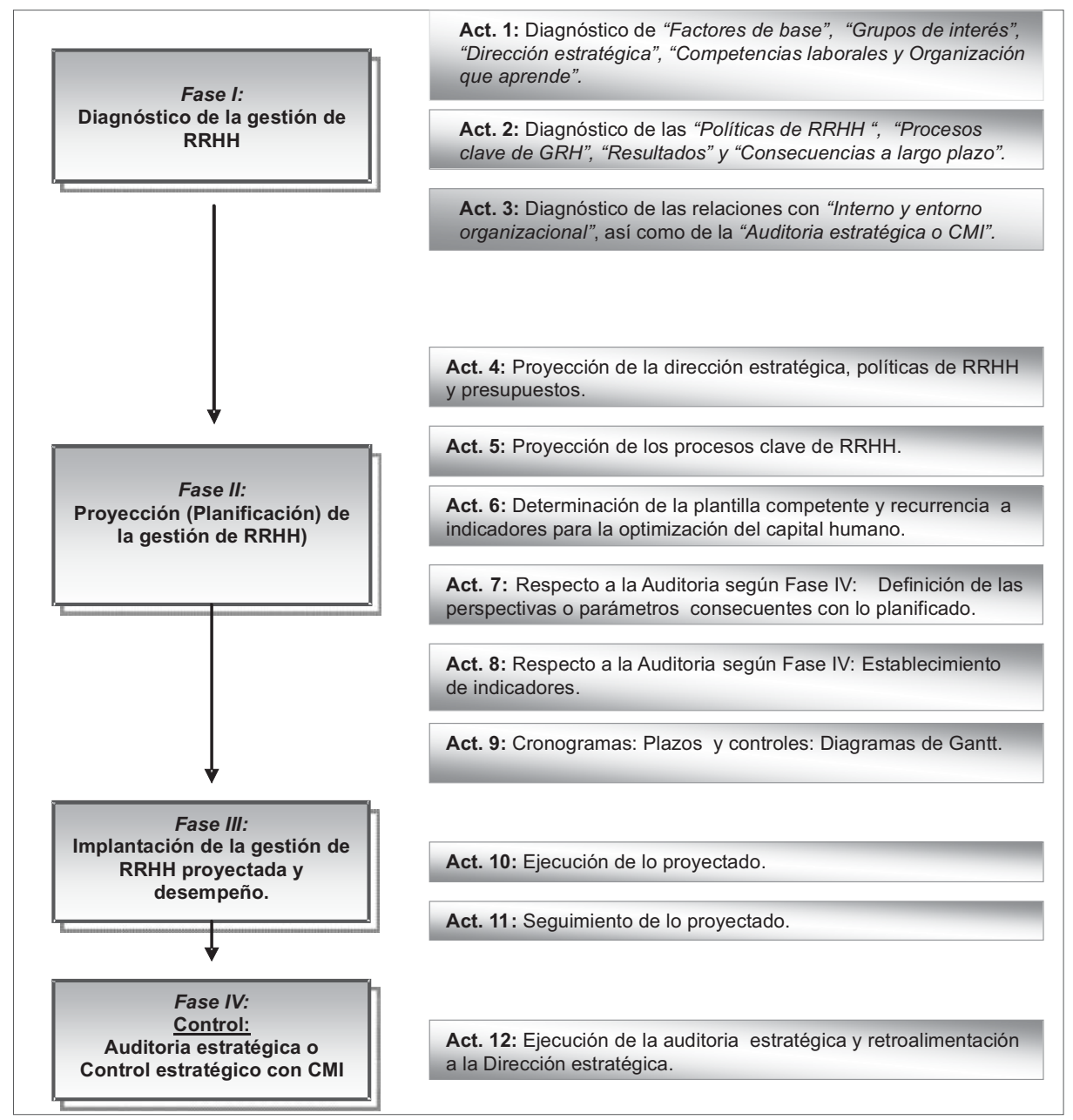

Fuente: Cuesta (2010).

la gestión de RRHH, ha sido impactada por las nuevas TIC, que en su búsqueda de la digitalización de la gestión de RRHH superó el tratamiento del capital humano comprendiendo intangibles de toda la or- ganización, ampliando el objeto de la gestión de RRHH al objeto de la gestión del conocimiento. En la contemporaneidad, por la consideración del entramado o la complejidad, es imprescindible apre- 
ciar de manera holistica o integrada esa GRH o gestión humana con la gestión del conocimiento, y todo ello alineado o coherente con la estrategia organizacional teniendo en cuenta las interacciones con el entorno extremadamente cambiante.

El modelo propuesto difiere de los consignados en la literatura científica actual al considerar el ciclo completo de la gestión del cambio, al comprender el diagnóstico, la planeación, el desempeño y el control estratégico de esa gestión, superando a los modelos predominantes que han sido de tipo descriptivo por este devenido funcional dada la tecnología implicada. El valor agregado esencial lo ofrece el aterrizaje que posibilita la referida tecnología. Además, la consideración integracionista de la gestión de recursos humanos con la gestión del conocimiento ofrece una contextualización con mayor sentido holístico que bien está demandando el pensamiento complejo de esta contemporaneidad.

Ese modelo integrando gestión humana y del conocimiento fue referente para el establecimiento de las normas cubanas (NC) sobre el "Sistema de Gestión Integrada de Capital Humano" -hoy en plena vigencia-, que se hayan refrendadas para el proceso de Perfeccionamiento Empresarial que comprende a todas las empresas cubanas.

\section{Referencias Bibliográficas}

Aguirre, Vivian (2001). "Análisis de GRH en el proceso de planeamiento técnico de la Red de Telecomunicaciones de ETECSA", Tesis de Maestría en opción al título de Master en Gestión de Recursos Humanos. La Habana, Ed. CUJAE.

Ballivián, Rafael (2006). “Desarrollo de un manual de selección de personal con un enfoque de proceso para la empresa Correos de Cuba", Tesis de Maestría en opción al título de Master en Gestión de Recursos Humanos, La Habana. Cuba, Ed. CUJAE.

Beer, Michael; Spector, Bert; Lawrence, Paul; Quinn, Daniels; Walton, Richard (1985). Human Resource Management. A General Manager's Perspective. Text and Cases, New York. USA. Free Press Macmillan.

Boxall, Peter, Purcell, John, \& Wright, Patrick (2007). "Human resource management: Scope, analysis and significance". In P. Boxall, J. Purcell, \& P. Wright (Eds.), The handbook of human resource management Oxford: Oxford University Press, pp. 1-16.

Chiavenato, Idalberto (2002). Gestión del talento humano, Bogotá. Colombia, Ed. Prentice Hall.

Cuesta, Armando (1999). Tecnología de gestión de recursos humanos. La Habana. Cuba. Ed. Academia.

Cuesta, Armando (2005). Tecnología de gestión de recursos humanos. La Habana. Cuba. Ed. Academia.

Cuesta, Armando (2010). Gestión del talento humano y del conocimiento, Bogotá. Colombia Ed. ECOE Ediciones.

Cuesta, Armando, Iliana Alcaide y Carlos López (2010). "Capital humano y eRRHH", en Revista Cubana de Ingeniería, Vol.1, No.1, 2010, Ed. ISPJAE, La Habana. Cuba. Pp. 25-32.

Cuesta, Armando, Silvio Viña, y Juan Marsán (1994). "Concepción de una maestría en gestión de recursos humanos", en revista Factores Humanos, de España, No. 4, Ed. Telefónica, Investigación y Desarrollo, Pp. 12-22. 
Dolan, Simon; Valle, Ramón; Jackson, Susan; Shuller, Randall (2003). La gestión de los recursos humanos, Madrid. España. Ed. McGraw-Hill.

Edvinsson, Lief y Michael Malone (1999): EI capital intelectual (cómo identificar y calcular el valor de los recursos intangibles de su empresa), Barcelona. España. Ed. Gestión 2000.

Fitz-eng, Jac (1999). Cómo medir la gestión de los recursos humanos, Bilbao. España. Ed. Deusto.

Fleury, Maria Teresa Leme y Fleury, Alfonso Carlos (2005). "In search of competence: aligning strategy and competences in the telecommunications industry", in International Journal of Human Resource Management, 16(9), 1640-1655, http://www. Tandfonline.com/doi/abs/10.1080/ $09585190500239275 \#$ preview, consultado en diciembre de 2011.

Gates, Bill (1999). Los negocios en la era digital, Barcelona. España. Ed. Plaza \& Janes.

Hernández, Irismaida (2003). "Los valores en una organización que aprende", Tesis de Maestría en opción al título de Master en Gestión de Recursos Humanos. La Habana. Cuba. Ed. CUJAE.

Kaplan, Robert \& David Norton (2004). Strategy maps: Converting intangible assets into tangible outcomes, Harvard, Ed. Harvard Business School Press.

Lengnick-Hall, Mark; Lengnick-Hall, Cynthia; Andrade, Leticia; Drake, Brian (2009). "Strategic human resource management: The evolution of the field", in Human Resource Management Review 19, pp.64-85, journal homepage: www.elsevier.com/locate/humres, consultado en marzo de 2010.

Lewin, Kurt (1948). Resolving Social Conflict, New York. USA. Ed. Harper \& Brothers.
Morales, Alfredo (2009). Capital humano (hacia un sistema de gestión en la empresa cubana). La Habana. Cuba. Ed. Política.

NC 3001: 2007. Norma Cubana NC 3001: 2007: "Sistema de gestión integrada de capital humano - Requisitos", 2007, La Habana. Cuba. Ed. Oficina Nacional de Normalización (NC), en www.nc.cubaindustria.cu, consultado en marzo de 2009.

Sánchez, Annia (2007). "Metodología para la planificación de los recursos humanos en la Sub-dirección de Proyectos del Instituto de Estudios e Investigaciones del Trabajo", Tesis de Maestría en opción al título de Master en Gestión de Recursos Humanos. La Habana. Cuba. Ed. CUJAE.

Senge, Peter et al. (1999). La quinta disciplina en la práctica. Barcelona. España. Ed. Granica.

Som, Ashok (2007). "What drives adoption of innovative SHRM practices in Indian organizations?", in International Journal of Human Resource Management, 18(5), pp. 808-828, journal homepage: www.elsevier.com/locate/humres, consultado en marzo de 2009.

Sveiby, Karl (2000). "O valor do intangivel”, em revista HSM Management, Ano 4, No.22, São Paulo. Brasil. Ed. Savana, pp.66-69.

Tristá, Grisel (2002). “El proceso de perfeccionamiento empresarial en marcha", en revista Nueva empresa, Vol.2, No.1. La Habana. Cuba. Ed. Empresa de Gestión del Conocimiento y la Tecnología (GECYT), pp.34-37.

Wei, Li-Qun, \& Lau, Chung-Ming (2005). "Market orientation, HRM importance and competency: Determinants of strategic HRM in Chinese firms", in International Journal of Human Resource Management, 16(10), pp. 1901- 
1918, Journal homepage: www.elsevier.com/locate/humres, consultado en marzo de 2009.

Ulrich, Davis (2006). "The RH Architect", en Aedipe, en http://www.aedipe.es/documentos/HRarchitect.doc, consultado en septiembre de 2006.
Werther, William y Keith Davis (2001). Administración de personal y recursos humanos. 5ta. Edición, México. México. Ed. Mc Graw Hill. 